\title{
Associations of beta-catenin alterations and MSI screening status with expression of key cell cycle regulating proteins and survival from colorectal cancer
}

Sakarias Wangefjord ${ }^{1 *}$, Jenny Brändstedt ${ }^{1}$, Kajsa Ericson Lindquist ${ }^{1}$, Björn Nodin ${ }^{1}$, Karin Jirström ${ }^{1}$ and Jakob Eberhard ${ }^{1,2}$

\begin{abstract}
Background: Despite their pivotal roles in colorectal carcinogenesis, the interrelationship and prognostic significance of beta-catenin alterations and microsatellite instability (MSI) in colorectal cancer (CRC) needs to be further clarified. In this paper, we studied the associations between beta-catenin overexpression and MSI status with survival from CRC, and with expression of p21, p27, cyclin D1 and p53, in a large, prospective cohort study.

Methods: Immunohistochemical MSI-screening status and expression of p21, p27 and p53 was assessed in tissue microarrays with tumours from 557 cases of incident CRC in the Malmö Diet and Cancer Study. Chi Square and Spearman's correlation tests were used to explore the associations between beta-catenin expression, MSI status, clinicopathological characteristics and investigative parameters. Kaplan-Meier analysis and Cox proportional hazards modelling were used to assess the relationship between beta-catenin overexpression, MSI status and cancer specific survival (CSS).

Results: Positive MSI screening status was significantly associated with older age, female sex, proximal tumour location, non-metastatic disease, and poor differentiation, and inversely associated with beta-catenin overexpression. Beta-catenin overexpression was significantly associated with distal tumour location, low T-stage and well-differentiated tumours. Patients with MSI tumours had a significantly prolonged CSS in the whole cohort, and in stage III-IV disease, also in multivariable analysis, but not in stage I-II disease. Beta-catenin overexpression was associated with a favourable prognosis in the full cohort and in patients with stage III-IV disease. Neither MSI nor beta-catenin status were predictive for response to adjuvant chemotherapy in curatively treated stage III patients. P53 and p27 expression was positively associated with beta-catenin overexpression and inversely associated with MSI. Cyclin D1 expression was positively associated with MSI and beta-catenin overexpression, and p21 expression was positively associated with MSI but not beta-catenin overexpression.
\end{abstract}

Conclusions: Findings from this large, prospective cohort study demonstrate that MSI screening status in colorectal cancer is an independent prognostic factor, but not in localized disease, and does not predict response to adjuvant chemotherapy. Beta-catenin overexpression was also associated with favourable outcome but not a treatment predictive factor. Associations of MSI and beta-catenin alterations with other investigative and clinicopathological factors were in line with the expected.

Virtual slides: The virtual slides for this article can be found here: http://www.diagnosticpathology.diagnomx.eu/vs/ 8778585058652609

\footnotetext{
* Correspondence: sakarias.wangefjord@med.lu.se

'Department of Clinical Sciences, Division of Pathology, Lund University,

Skåne University Hospital, Lund 221 85, Sweden

Full list of author information is available at the end of the article
} 


\section{Background}

Colorectal cancer (CRC) is the third most common cancer in men and the second most common cancer in women worldwide. More than 1,2 million new cases are diagnosed globally every year, with more than 600,000 related deaths in 2008 [1].

Colorectal carcinogenesis is a complex multistage process involving multiple genetic alterations and at least three distinct pathogenetic pathways have been characterized: chromosomal instability (CIN), microsatellite instability and $\mathrm{CpG}$ island methylator phenotype (CIMP) [2,3].

The chromosomal instability pathway (CIN) is characterized by karyotypic abnormalities, e.g. aneuploidy and loss of heterozygosity, and certain mutations including activation of K-ras and inactivation of APC, loss of p53, and loss of heterozygosity for the long arm of chromosome 18 . This pathway accounts for $65-70 \%$ of sporadic CRC $[4,5]$ and for cancers associated with familial adenomatous polyposis (FAP), constituting less than $1 \%$ of all CRC [6].

Beta-catenin is a membrane-associated protein with essential functions in the regulation of cellular adhesion and as the major mediator of the Wnt-signaling pathway. When Wnt-receptors are activated, kinases in the APC complex are inhibited, leading to accumulation of cytoplasmic beta-catenin and its translocation to the nucleus. This facilitates the transcription of various target genes, e.g. cyclin D1, hence contributing to tumour progression [7-11].

Despite its crucial role in colorectal carcinogenesis, previous studies on altered beta-catenin expression as a prognostic marker or predictor of chemotherapy response in CRC have been conflicting [12-15].

The second pathway is initiated by germline mutations in the mismatch repair (MMR) genes, e.g. MLH1, MSH2, MSH6, and PMS2, or somatic tumour MLH1 promoter methylation, leading to microsatellite instability (MSI). MSI is detected in $15-20 \%$ of all cases of CRC, predominantly tumours located in the proximal colon, and in almost all tumours from patients with hereditary nonpolyposis colon cancer (HNPCC), accounting for 3$5 \%$ of all CRC $[16,17]$.

CRC patients with MSI tumours seem to have a significantly better prognosis compared to those with MSS in most studies, but might not benefit from adjuvant fluorouracil (FU) treatment [16-22]. On a molecular level, there is in vitro data supporting that an intact mismatch repair system is required to induce apoptosis of FU-modified DNA [23].

Finally, the CpG island methylator phenotype (CIMP) provides a third pathway to CRC through predisposition to epigenetic DNA hypermethylation, causing transcriptional silencing of tumour supressor genes [24,25].
The aim of the present study was to examine the prognostic and treatment predictive significance of betacatenin overexpression and MSI screening status in tumours from a large prospective CRC cohort $(n=557)$. A secondary aim was to examine the associations of beta-catenin alterations and MSI status with key cell cycle regulators p53, p21, p27 and cyclin D1. In a previous study on tumours from this cohort, elevated cyclin D1 expression was found to associate with a prolonged survival, in particular in male patients [26].

\section{Methods}

\section{Study group}

The Malmö Diet and Cancer Study (MDCS) is a population-based prospective cohort study with the primary aim to examine whether a Western diet rich in fat and low in fruit and vegetables increases the risk of certain forms of cancer. Between 1991-1996, a total number of 30446 individuals; 12120 (39.8\%) men and 18 $326(60.2 \%)$ women between 44-74 years where enrolled (from a background population of 74 138) [27]. Until 31 Dec 2008, 626 incident cases of CRC had been registered in the MDCS. Cases were identified from the Swedish Cancer Registry up until 31 Dec 2007, and from The Southern Swedish Regional Tumour Registry for the period of 1 Jan - 31 Dec 2008. All tumours with available slides or paraffin blocks were histopathologically reevaluated on haematoxylin and eosin stained slides. Histopathological, clinical and treatment data were obtained from the clinical and/or pathology records. TNM staging was performed according to the American Joint Committee on Cancer (AJCC). Information on vital status and cause of death was obtained from the Swedish Cause of Death Registry up until 31 Dec 2009. Followup started at date of diagnosis and ended at death, emigration or 31 Dec 2009, whichever came first. None of the CRC cases registered until 31 Dec 2008 was lost due to emigration during follow-up. Median follow-up time was 3.35 years (range $0-17.69)$ for the full cohort $(\mathrm{n}=626)$ and 6.05 years (range 1.03-17.69) for patients alive $(n=344)$. Patient and tumour characteristics of the cohort have been described in detail previously $[26,28,29]$. Ethical permission was obtained from the Ethics Committee at Lund University for the MDCS (Ref. 51/90), and the present study (Ref. 530/2008).

\section{Tissue microarray construction}

Cases with an insufficient amount of tumour material were excluded, whereby a total number of 557 (89.0\%) tumours were suitable for tissue microarray (TMA) construction. Areas representative of cancer were marked on haematoxylin \& eosin stained slides and TMAs were constructed as previously described $[26,28]$. In brief, two $1.0 \mathrm{~mm}$ cores were taken from each tumour and 

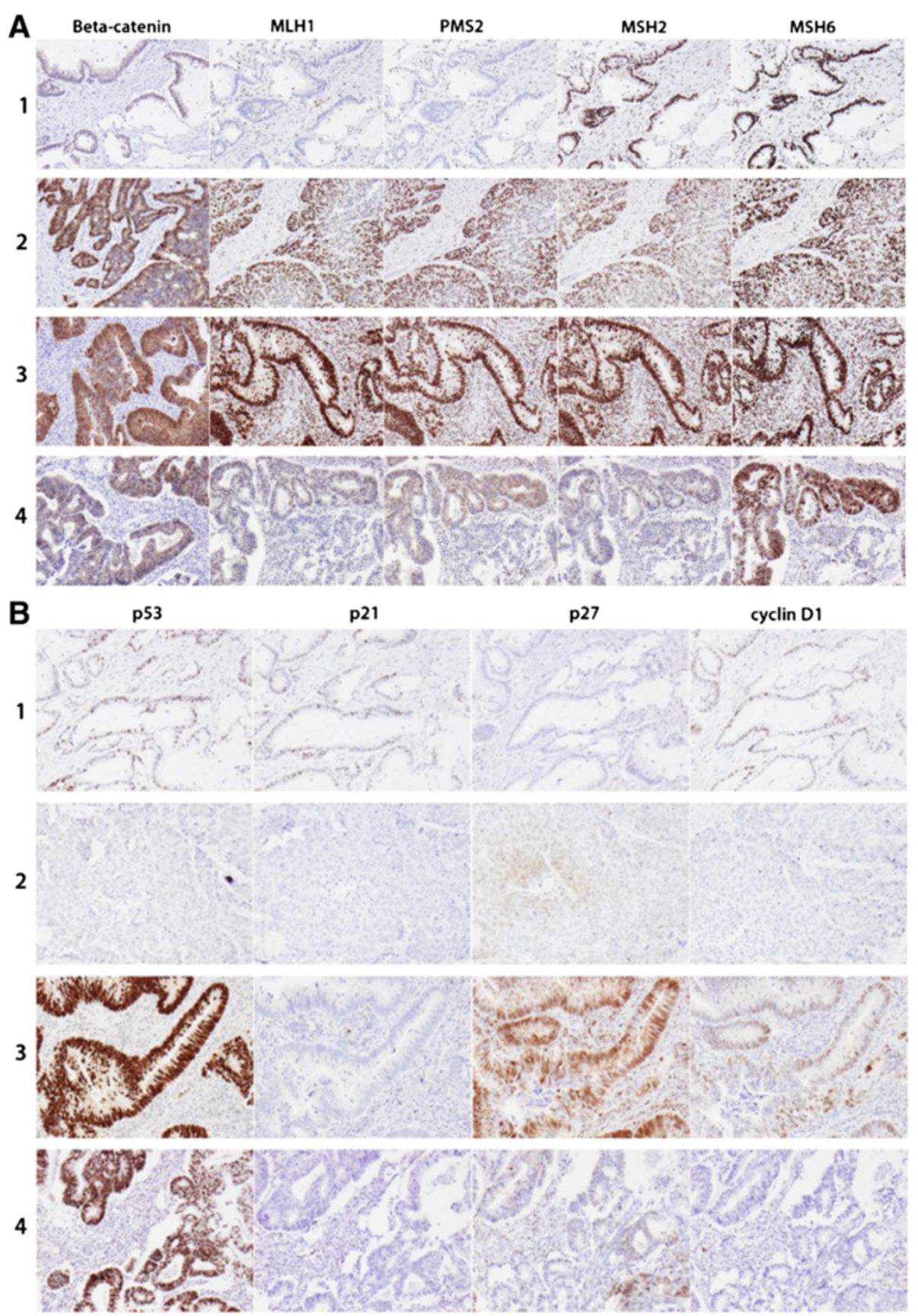

Figure 1 Distribution of the investigative markers in four cases. Immunohistochemical images from four different cases. 1. Female patient, age 70, with T3NOMO tumour in transverse colon; beta-catenin grade 1 (0-1-0), MSI, p53 negative, p21 = 2/1, p27 negative, cyclin D1 = 2/2. 2: Female patient, age 77, with T4N1M1 tumour in descendent colon; beta-catenin grade 3 (0-1-2), MSS, p53 negative, p21 negative, p27 2/2, cyclin D1 1/1. 3. Male patient, age 75, with T4NXM tumour in proximal colon; beta-catenin grade 5 (1-2-2), MSS, p53 positive, p21 negative, p27 4/3, cyclin D1 3/2. 4. Male patient, age 68, with T2N2M0 rectal cancer; beta-catenin grade 3 (1-1-1), MSS; p53 positive, p21 negative, p27 3/3, cyclin D1 negative. A: staining of beta-catenin (membrane-cytoplasm-nucleus) and mismatch repair proteins MLH1, PMS2, MSH2 and MSH6. B: staining of p53, p21 (fraction/intensity), p27 (fraction/intensity) and cyclin D1 (fraction/intensity). 
Table 1 Associations of MSI screening status and beta-catenin expression with clinicopathological characteristics

\begin{tabular}{|c|c|c|c|c|c|c|c|}
\hline \multirow{3}{*}{ n (\%) } & \multicolumn{3}{|c|}{ MSI screening status } & \multicolumn{3}{|c|}{ Beta-catenin score } & \multirow{3}{*}{ P-value } \\
\hline & MSS & MSI & $\overline{P \text {-value }}$ & $0-1$ & $2-3$ & $4-5$ & \\
\hline & $438(85.0)$ & $77(15.0)$ & & $159(30.2)$ & $170(32.3)$ & $198(37.6)$ & \\
\hline \multicolumn{8}{|l|}{ Age } \\
\hline$<=75$ & 308(70.3) & $41(53.2)$ & $0.003^{*}$ & $101(63.5)$ & 123(72.4) & 133(67.2) & 0.561 \\
\hline$>75$ & 130(29.7) & $36(46.8)$ & & $58(36.5)$ & $47(27.6)$ & $65(32.8)$ & \\
\hline \multicolumn{8}{|l|}{ Sex } \\
\hline Female & $222(50.7)$ & $50(64.9)$ & $0.021^{*}$ & $87(54.7)$ & $90(52.9)$ & $100(50.5)$ & 0.425 \\
\hline Male & $216(49.3)$ & $27(35.1)$ & & $72(45.3)$ & $80(47.1)$ & $98(49.5)$ & \\
\hline \multicolumn{8}{|c|}{ Tumour location } \\
\hline Proximal & 107(24.5) & $62(80.5)$ & $<0.001^{* *}$ & $84(53.2)$ & $50(29.4)$ & $37(18.8)$ & $<0.001^{* *}$ \\
\hline Transverse & $12(2.8)$ & $8(10.4)$ & & $11(7.0)$ & $7(4.1)$ & $5(2.5)$ & \\
\hline Descending & $26(6.0)$ & $1(1.3)$ & & $6(3.8)$ & $11(6.5)$ & $9(4.6)$ & \\
\hline Sigmoid & 105(24.1) & $3(3.9)$ & & 19(12.0) & $31(18.2)$ & $60(30.5)$ & \\
\hline Rectum & 186(42.7) & $3(3.9)$ & & $38(24.1)$ & $71(41.8)$ & $86(43.7)$ & \\
\hline Unknown & 2 & 0 & & 1 & 0 & 1 & \\
\hline \multicolumn{8}{|l|}{ T stage } \\
\hline 1 & $45(10.8)$ & $3(3.9)$ & 0.116 & $12(8.0)$ & $11(6.9)$ & 23(11.9) & $0.039^{*}$ \\
\hline 2 & $53(12.7)$ & $7(9.2)$ & & $14(9.3)$ & $21(13.1)$ & $27(14.0)$ & \\
\hline 3 & $257(61.5)$ & $54(71.1)$ & & $99(66.0)$ & $100(62.5)$ & $119(61.7)$ & \\
\hline 4 & 63(15.1) & 12(15.8) & & $25(16.7)$ & $28(17.5)$ & $24(12.4)$ & \\
\hline missing & 20 & 1 & & 9 & 10 & 5 & \\
\hline \multicolumn{8}{|l|}{$\mathrm{N}$ stage } \\
\hline 0 & $222(56.5)$ & $54(72.0)$ & $0.017^{*}$ & $84(57.5)$ & $86(55.8)$ & 109(60.9) & 0.249 \\
\hline 1 & $101(25.7)$ & 12(16.0) & & 28(19.2) & $44(28.6)$ & $44(24.6)$ & \\
\hline 2 & $70(17.8)$ & $9(12.0)$ & & $34(23.3)$ & 24(15.6) & $26(14.5)$ & \\
\hline missing & 45 & 2 & & 13 & 16 & 19 & \\
\hline \multicolumn{8}{|l|}{ M stage } \\
\hline 0 & $352(81.7)$ & $72(93.5)$ & $0.010^{*}$ & 123(78.8) & 140(83.3) & 166(85.1) & 0.131 \\
\hline 1 & $79(18.3)$ & $5(6.5)$ & & $33(21.2)$ & $28(16.7)$ & $29(14.9)$ & \\
\hline missing & 7 & 0 & & 3 & 2 & 3 & \\
\hline \multicolumn{8}{|c|}{ Differentiation grade } \\
\hline High & $28(6.5)$ & $6(8.0)$ & $0.003^{* *}$ & $16(10.3)$ & $8(4.8)$ & $10(5.1)$ & $0.016^{*}$ \\
\hline Intermediate & $321(74.5)$ & 40(53.3) & & $94(60.3)$ & 115(69.3) & 159(81.5) & \\
\hline Low & $82(19.0)$ & $29(38.7)$ & & $46(29.5)$ & $43(25.9)$ & $26(13.3)$ & \\
\hline missing & 7 & 2 & & 3 & 4 & 4 & \\
\hline \multicolumn{8}{|c|}{ Vascular invasion } \\
\hline No & $121(47.8)$ & 26(57.8) & 0.220 & $48(50.5)$ & $33(36.3)$ & $68(58.1)$ & 0.177 \\
\hline Yes & $132(52.2)$ & 19(42.2) & & $47(49.5)$ & $58(63.7)$ & 49(41.9) & \\
\hline missing & 185 & 32 & & 64 & 79 & 81 & \\
\hline
\end{tabular}

Columnar percentage in parentheses. $\mathrm{x} 2$-test of association was used for $2 \times 2$ tables and $\mathrm{x} 2$-test for linear trend for tables with more than two rows and/or columns. The categories marked as missing were not included in the analysis. *Significant at the 0.05 level. * Significant at the 0.01 level. N1 $=1-3$ positive nodes, $\mathrm{N} 2=>4$ positive nodes. $\mathrm{MSS}=$ Microsatellite stable, MSI = Microsatellite unstable. Proximal colon = appendix, cecum, ascending colon, hepatic flexure; descending colon = splenic flexure and descending colon. 
mounted in a new recipient block using a semiautomated arraying device (TMArrayer, Pathology Devices, Westminster, MD, USA). As demonstrated previously, there was no selection bias regarding the distribution of clinicopathological characteristics between the TMA cohort and the full cohort [28].

\section{Immunohistochemistry and staining evaluation}

For immunohistochemical analysis, $4 \mu \mathrm{m}$ TMA-sections were transferred to glass slides (Menzel Super Frost Plus), dried at room temperature, and baked in a heated chamber for 2 hours at $60^{\circ} \mathrm{C}$. Deparaffinization and antigen retrieval was performed using the PT Link system (DAKO, Glostrup, Denmark) in a high pH buffer. Staining was performed in an Autostainer Plus (DAKO) with monoclonal antibodies against p21 (clone SX118, DAKO) diluted 1:25, p27 (clone SX53G8, DAKO) diluted 1:100, and p53 (clone DO-7, DAKO) diluted 1:100, using a diamino-benzidine (DAB) based visualization kit (K801021-2, Dako Denmark A/S). Counterstaining was performed using Mayer's hematoxylin. Immunohistochemical staining and evaluation of cyclin D1 expression had been performed previously using a monoclonal anti-cyclin D1 antibody DSC-6 (DAKO) diluted 1:50 [26]. Nuclear expression of p21, p27 and cyclin D1 was evaluated in a similar fashion, taking both the fraction of positive cells and staining intensity into account. Specifically, the nuclear staining intensity was denoted as 0 (negative), 1 (weak), 2 (moderate) or 3 (strong), and the proportion of positive tumour nuclei as 0 (0 to $1 \%$ ), 1 ( 2 to $25 \%$ ), 2 (26 to $50 \%$ ), 3 (51 to $75 \%$ ) and 4 (> 75\%). p53 positivity was defined as > = 50\% tumour cells with strong nuclear staining intensity in acordance with previous studies [30].

For definition of MSI screening status, immunohistochemical staining for MLH1, PMS2, MSH2 and MSH6 was performed as previously described [29]. Immunohistochemical stainings were evaluated as negative when all tumour cells showed loss of nuclear staining. Surrounding stromal cells and tumour infiltrating lymphocytes served as internal controls for each biopsy core. A nuclear reaction of tumour cells was assessed as a positive staining. MSI screening status was evaluated using monoclonal antibodies against MLH1 (Clone ES05, DAKO, diluted 1 : 100), PMS2 (Clone A16-4, 556415, BD Pharmingen, San Diego, CA, USA, diluted 1 : 300), MSH2 (Clone FE11, NA27, Calbiochem, San Diego, CA, USA, diluted 1 : 100), and MSH6 (EPR3945, Epitomics, Burlingame, CA, USA, diluted $1: 100)$ as previously described [31]. Immunohistochemical stainings were evaluated as negative when all tumour cells showed loss of nuclear staining. Surrounding stromal cells and tumour infiltrating lymphocytes served as internal controls for each biopsy core. Any nuclear reaction of tumour cells was assessed as positive staining. Tumour samples lacking nuclear staining of MLH1, PMS2, MSH2 or MSH6 were considered to have a positive MSI screening status, and referred to as MSI, and tumours with negative MSI screening status are referred to as MSS.

Immunohistochemical staining of beta-catenin was performed with a monoclonal anti-beta-catenin antibody (\# 610153 BD Pharmingen, San Diego, Ca, USA), diluted 1:5000. The staining was evaluated as previously described [32,33], whereby membranous staining was denoted as 0 (present) or 1 (absent), cytoplasmic staining intensity as $0-2$ and nuclear staining intensity as $0-2$. The total score ranging from 0 (corresponding to membrane staining only, as in normal colonic mucosa) to 5 (tumours with strong nuclear and cytoplasmic staining) was then divided into three categories; $1=$ score $0-1$, $2=$ score $2-3$ and $3=$ score $4-5$ ).

All immunohistochemical stainings were evaluated by two independent observers (SW and $\mathrm{KJ}$ ), who were blinded to clinical and outcome data. Scoring differences were discussed in order to reach consensus.

\section{Statistical analysis}

Chi Square and Spearman's correlation (R) tests were used to explore the associations between clinicopathological and investigative parameters. Kaplan-Meier analysis and log rank test were used to illustrate differences in cancer specific survival (CSS) according to beta-catenin overexpression and MSI screening status. Cox regression proportional hazards models were used for estimation of hazard ratios (HRs) for death from CRC in both uni- and multivariable analysis, adjusted for age, gender, T-stage, $\mathrm{N}$-stage, M-stage, differentiation grade and vascular invasion. A backward conditional selection method was used for variable selection by the model. All tests were twosided. A p-value of 0.05 was considered significant. All statistical analyses were performed using IBM SPSS Statistics version 20 .

\section{Results}

Associations of MSI screening status and beta-catenin overexpression with clinicopathological characteristics and investigative factors

Representative immunohistochemical images of the investigative markers in tumours from four different cases are shown in Figure 1A \& 1B.

As seen in Table 1 there were significant associations between MSI and older age, female sex, proximal tumour location, non metastatic disease (N0,M0) and low differentiation grade. Further, beta-catenin expression was associated with distal tumour location, less advanced T-stage and intermediate or high tumour differentiation grade.

Associations between beta-catenin overexpression, MSI screening status and investigative factors are shown 
Table 2 Associations of MSI screening status and beta-catenin expression with expression of p21, p27 and p53

\begin{tabular}{|c|c|c|c|c|c|c|c|}
\hline \multirow{3}{*}{ n (\%) } & \multicolumn{3}{|c|}{ MSI screening status } & \multicolumn{3}{|c|}{ Beta-catenin score } & \multirow{3}{*}{$P$-value } \\
\hline & MSS & MSI & P-value & $0-1$ & $2-3$ & 4-5 & \\
\hline & $438(85.0)$ & $77(15.0)$ & & $159(30.2)$ & $170(32.3)$ & 198(37.6) & \\
\hline \multicolumn{8}{|l|}{ P53 status } \\
\hline Negative & 196(40.0) & $62(80.5)$ & $<0.001^{* *}$ & $101(65.2)$ & $92(55.1)$ & $76(38.6)$ & $<0.001^{* *}$ \\
\hline Positive & 230(54.0) & $15(19.5)$ & & $54(34.8)$ & $75(44.9)$ & $121(61.4)$ & \\
\hline missing & 12 & 0 & & 4 & 3 & 1 & \\
\hline \multicolumn{8}{|c|}{ Cyclin D1 fraction } \\
\hline $0-1 \%$ & $94(22.1)$ & $2(2.6)$ & $<0.001^{* *}$ & $36(23.4)$ & $36(21.7)$ & $31(15.8)$ & $0.001^{* *}$ \\
\hline $2-25 \%$ & 159(37.4) & $26(34.2)$ & & $63(40.9)$ & $65(39.2)$ & 63(32.1) & \\
\hline $26-50 \%$ & $65(15.3)$ & $16(21.1)$ & & $20(13.0)$ & $29(17.5)$ & $33(16.8)$ & \\
\hline $51-75 \%$ & $87(20.5)$ & 25(32.9) & & $27(17.5)$ & $32(19.3)$ & $53(27.0)$ & \\
\hline$>75 \%$ & $20(4.7)$ & $7(9.2)$ & & $8(5.2)$ & $4(2.4)$ & $16(8.2)$ & \\
\hline missing & 13 & 1 & & 5 & 4 & 2 & \\
\hline \multicolumn{8}{|c|}{ Cyclin D1 intensity } \\
\hline Negative & $94(22.1)$ & $2(2.6)$ & $<0.001^{* *}$ & $36(23.4)$ & $36(21.7)$ & $31(15.8)$ & 0.085 \\
\hline Weak & 154(36.2) & $20(26.3)$ & & $51(33.1)$ & $59(35.5)$ & $68(34.7)$ & \\
\hline Moderate & 129(30.4) & $41(53.9)$ & & 49(31.8) & $55(33.1)$ & $68(34.7)$ & \\
\hline Strong & 48(11.3) & $13(17.1)$ & & 18(11.7) & $16(9.6)$ & $29(14.8)$ & \\
\hline missing & 13 & 1 & & 5 & 4 & 2 & \\
\hline \multicolumn{8}{|l|}{ P27 fraction } \\
\hline $0-1 \%$ & $54(12.7)$ & $24(31.2)$ & $0.003^{* *}$ & $43(27.9)$ & 33(19.8) & $8(4.1)$ & $<0.001^{* *}$ \\
\hline $2-25 \%$ & $96(22.6)$ & $14(18.2)$ & & $36(23.4)$ & $43(25.7)$ & $35(17.9)$ & \\
\hline $26-50 \%$ & $90(21.2)$ & $16(20.8)$ & & $32(20.8)$ & $40(24.0)$ & $36(18.4)$ & \\
\hline $51-75 \%$ & 134(31.5) & $15(19.5)$ & & $31(20.1)$ & $36(21.6)$ & $83(42.3)$ & \\
\hline$>75 \%$ & $51(12.0)$ & $8(10.4)$ & & $12(7.8)$ & $15(9.0)$ & $34(17.3)$ & \\
\hline missing & 13 & 1 & & 5 & 3 & 2 & \\
\hline \multicolumn{8}{|c|}{ P27 intensity } \\
\hline Negative & $54(12.7)$ & $24(31.2)$ & $<0.001^{* *}$ & 43/27.9) & $33(19.8)$ & $8(4.1)$ & $<0.001^{* *}$ \\
\hline Weak & $96(22.6)$ & $22(28.6)$ & & $46(29.9)$ & $39(23.4)$ & $37(18.9)$ & \\
\hline Moderate & 142(33.4) & $18(23.4)$ & & $50(32.5)$ & $51(30.5)$ & $62(31.6)$ & \\
\hline Strong & 133(31.3) & 13(16.9) & & $15(9.7)$ & $44(26.3)$ & $89(45.4)$ & \\
\hline missing & 13 & 1 & & 5 & 3 & 2 & \\
\hline \multicolumn{8}{|l|}{ P21 fraction } \\
\hline $0-1 \%$ & $69(16.3)$ & $3(3.9)$ & $0.002^{* *}$ & $25(16.2)$ & $27(16.2)$ & $25(12.9)$ & 0.835 \\
\hline $2-25 \%$ & 233(55.1) & $41(53.2)$ & & 81(52.6) & $90(53.9)$ & 112857.7) & \\
\hline $26-50 \%$ & $60(14.2)$ & 18(23.4) & & 24(15.6) & $28(16.8)$ & $26(13.4)$ & \\
\hline $51-75 \%$ & $53(12.5)$ & 13(16.9) & & 21(13.6) & $16(9.6)$ & $30(15.5)$ & \\
\hline$>75 \%$ & $8(1.9)$ & $2(2.6)$ & & $3(1.9)$ & $6(3.6)$ & $1(0.5=$ & \\
\hline missing & 15 & 1 & & 5 & 3 & 4 & \\
\hline \multicolumn{8}{|c|}{ P21 intensity } \\
\hline Negative & $69(16.3)$ & $3(3.9)$ & $<0.001^{* *}$ & $25(16.2)$ & $27(16.2)$ & $25(12.9)$ & 0.567 \\
\hline Weak & 174(41.1) & 24(31.2) & & $60(39.0)$ & $63(37.7)$ & $81(41.8)$ & \\
\hline Moderate & $122(28.8)$ & $36(46.8)$ & & $51(33.1)$ & $52(31.1)$ & $60(30.9)$ & \\
\hline
\end{tabular}


Table 2 Associations of MSI screening status and beta-catenin expression with expression of p21, p27 and p53 (Continued)

\begin{tabular}{|c|c|c|c|c|c|c|}
\hline Strong & $58(13.7)$ & 14(18.2) & $18(11.7)$ & $25(15.0)$ & $28(14.4)$ & \\
\hline missing & 15 & 1 & 5 & 3 & 4 & \\
\hline \multicolumn{7}{|c|}{ MSI screening status } \\
\hline MSS & - & - & 109(72.7) & 136(83.4) & 184(95.8) & \multirow[t]{3}{*}{$<0.001^{* *}$} \\
\hline MSI & - & - & $41(27.3)$ & $27(16.6)$ & $8(4.2)$ & \\
\hline missing & & & 9 & 7 & 6 & \\
\hline
\end{tabular}

in Table 2. MSI screening status was inversely associated with beta-catenin overexpression. MSI correlated significantly to cyclin D1 expression (fraction and intensity), p21 expression (fraction and intensity), and inversely with p53 expression and p27 expression (fraction and intensity). Beta-catenin overexpression correlated significantly to p53 expression, cyclin D1 expression (fraction), and p27 expression (fraction and intensity).

\section{Association of MSI screening status and beta-catenin overexpression with survival}

Kaplan-Meier analysis showed significant correlations between MSI and prolonged CSS in the whole cohort (Figure 2A), and in stage III-IV disease (Figure 2E) but not in stage I-II disease (Figure 2C). Beta-catenin overexpression correlated significantly to a prolonged CSS in stage III-IV disease only (Figure 2F).

Uni- and multivariable (adjusted for age, T-stage, Nstage, $\mathrm{M}$-stage, differentiation grade and vascular invasion) Cox regression analysis confirmed the significant correlation between MSI and an improved CSS, both in the full cohort and in stage III-IV disease (Table 3). Beta-catenin overexpression was also associated with an improved CSS, in the full cohort and patients with stage III-IV disease, although these associations were less significant than for MSI status (Table 3). Notably, while only reaching statistical significance in multivariable analysis, the HRs for beta-catenin overexpression should be considered as being similar in univariable and multivariable analysis ( 0.75 vs 0.70 in the full cohort and 0.73 vs 0.67 in stage III-IV disease).

Next, we examined the potential treatment predictive value of MSI screening status and beta-catenin overexpression. Subgroup analysis of stage III disease patients in strata according to adjuvant treatment (no treatment, 5 -fluorouracil + leucovorin or 5-fluorouracil + leucovorin + oxaliplatin) revealed no significant correlations of MSI or beta-catenin overexpression and CSS (Figure 3).

The prognostic and treatment predictive value of MSI and beta-catenin overexpression did not differ according to gender (data not shown). Similar trends for the prognostic and treatment impact of MSI status and beta-catenin expression were seen for 5 -year overall survival (data not shown).

\section{Discussion}

In this study we have demonstrated that positive MSI screening status is associated with a more favourable prognosis and certain clinicopathological characteristics of CRC, i.e. older age, female sex, proximal tumour location and low differentiation grade. This is accordant to previous studies [16,17], thus consolidating our data. The correlation between MSI and a prolonged CSS was significant in both uni- and multivariable analysis for the whole cohort and for stage III-IV disease, but not for stage I-II disease. A possible interpretation of this observation could be that MSI reflects a less agressive phenotype, with an improved CSS even in the presence of metastatic disease and despite adverse pathological characteristics such as low differentiation grade.

Here, we could not demonstrate any association between MSI status and response to adjuvant treatment in curatively treated patients with stage III disease. On one hand, the subgroups available for analysis of the effects of adjuvant treatment were rather small, thus limiting statistical power. On the other hand, since this study started already in the mid 90s, when adjuvant chemotherapy was not yet standard of care in Sweden, nearly half of the curatively treated patients with stage III disease did not receive adjuvant treatment. Although treatment predictive markers are best evaluated in a controlled, prospective setting, the value of retrospective analyses on tumours from less extensively treated patients should not be underestimated.

Moreover, the feasibility of MSI as a predictor of therapeutic response has been debated, and a recent meta-analysis showed results concordant with ours, i.e. no treatment predictive value of MSI status [34].

Beta-catenin overexpression was also associated with a reduced risk of death in the full cohort and in patients with stage III-IV disease, although these associations were weaker than for MSI status. Previous studies on 

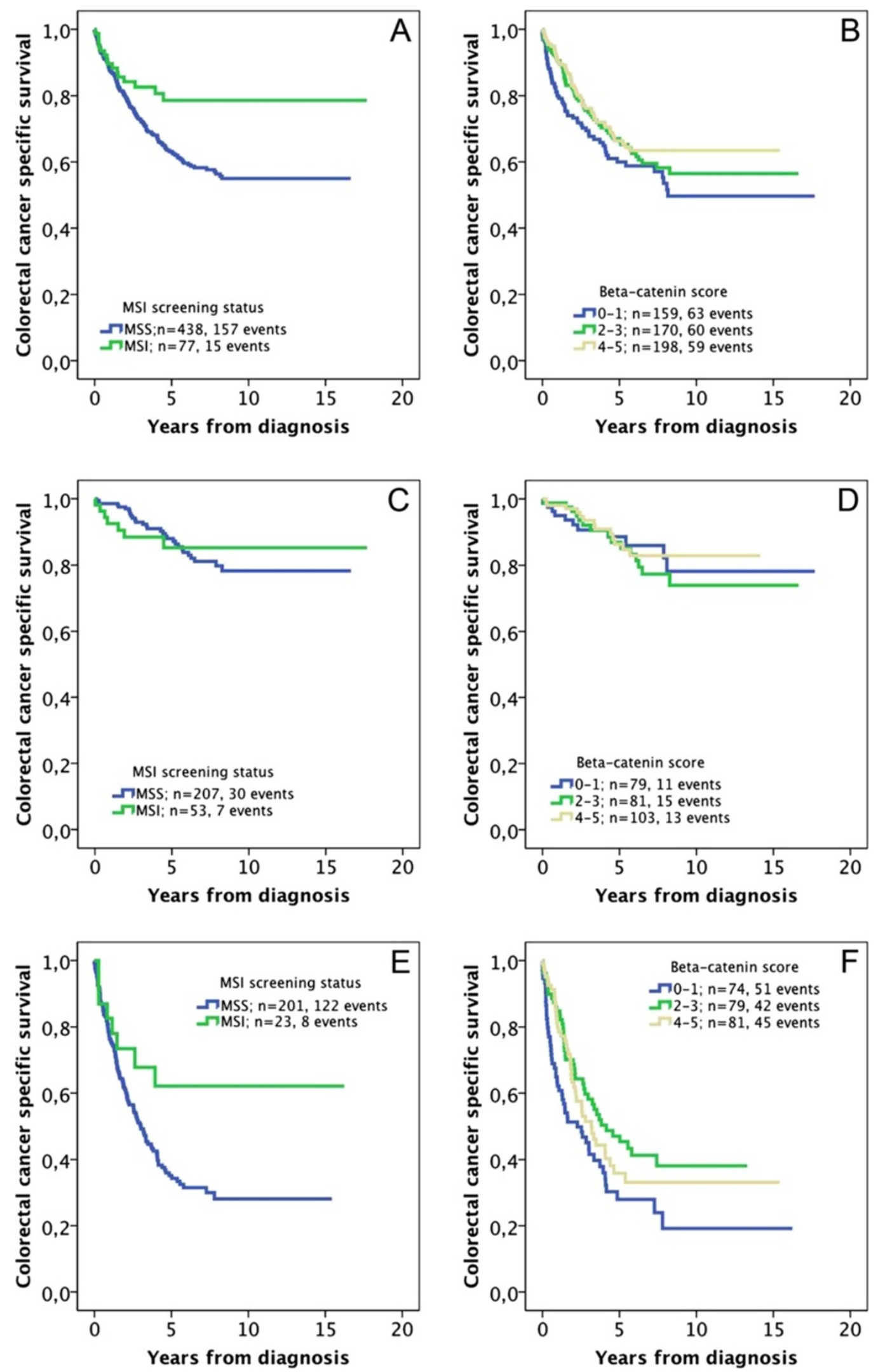

Figure 2 (See legend on next page.) 
(See figure on previous page.)

Figure 2 Associations between MSI screening status, beta-catenin overexpression and survival in all patients and in subgroups according to disease stage. Kaplan-Meier analysis of colorectal cancer specific survival according to: (A) MSI screening status in the full cohort (logrank $\left.p=0.008^{* *}\right)$, (B) Beta-catenin score in the full cohort (logrank $\left.p=0.116\right)$, (C) MSI screening status in stage I-II disease (logrank $\left.p=0.985\right)$, (D) Beta-catenin score in stage I-II disease (logrank p =0.725), (E) MSI screening status in stage III-IV disease (logrank $\left.p=0.032^{*}\right)$ and $(\mathbf{F})$ Betacatenin score in stage III-IV disease (logrank $\left.p=0.034^{*}\right)$.

beta-catenin as a prognostic marker have been conflicting, with some studies reporting a favourable or no prognostic value [14,35-38] and some an association with poor clinical outcome $[12,13,39,40]$. Many factors could explain the differing results regarding the prognostic value of beta-catenin overexpression; e.g. intrinsic tumour heterogeneity [41], different immunohistochemical staining and visualization methods with varying degrees of sensitivity, and lack of standardization of what constitutes a "positive" or "negative" result. In many cases, nuclear beta-catenin expression is predominantly seen in the margin and not in the center of the tumour [42]. Horst et al. postulated that lost beta-catenin regulation is presented rather as an altered intratumoral distribution of nuclear beta-catenin expression [43] and, in light of this observation, the TMA-technique may not be an ideal tool for its assessment. Moreover, the degree and prognostic implications of beta-catenin activation may differ in primary and metastatic CRC [44].

The herein observed inverse association between MSI and beta-catenin overexpression is also in accordance with previous studies $[45,46]$. Assuming that MSI status and beta-catenin overexpression represent two different pathways to colorectal tumorigenesis, it might be perceived as contradictory that both reflect an improved
CSS. However, as these pathways are not mutually exclusive and likely overlap, their complex interactions need further study.

The associations between MSI and cell cycle regulators cyclin D1 and p21 have been described previously $[47,48]$ but the underlying mechanisms still remain unclear. Similarly, the inverse associations of MSI tumours with p53 mutation status and p27 expression are well known $[30,48]$. The positive correlation between betacatenin overexpression and p53 status is also in line with previous studies $[14,39,45]$.

The observation of a positive association of cyclin D1 expression with MSI screening status and beta-catenin overexpression, respectively, is in line with the previously demonstrated findings of cyclin D1 expression being associated with a prolonged CSS [26]. Notably, the beneficial prognostic value of high cyclin D1 expression was only evident in male and not in female CRC patients [26]. In this study, the prognostic and treatment predictive value of MSI and beta-catenin overexpression did not differ according to sex.

Since the MDCS is a population-based cohort study, a potential selection bias compared with the general population must be taken into consideration, but the distribution of TNM-stages at diagnosis is in line with the

Table 3 Cox proportional hazards model for colorectal cancer specific survival related to MSI screening status and beta-catenin overexpression in the full cohort and in stage III-IV disease

\begin{tabular}{|c|c|c|c|c|c|c|}
\hline & \multicolumn{3}{|c|}{ Full cohort } & \multicolumn{3}{|c|}{ Stage III-IV disease } \\
\hline & $\mathrm{HR}(95 \% \mathrm{Cl})$ & $p$-value & $N($ events) & $\mathrm{HR}(95 \% \mathrm{Cl})$ & $p$-value & $n$ (events) \\
\hline & \multicolumn{3}{|c|}{ Univariable } & \multicolumn{3}{|c|}{ Univariable } \\
\hline MSS & 1,00 & $0,010^{* *}$ & 438(157) & 1,00 & $0,036^{*}$ & 201(122) \\
\hline \multirow[t]{2}{*}{ MSI } & $0,50(0,29-0,84)$ & & $77(15)$ & $0,46(0,23-0,95)$ & & $23(8)$ \\
\hline & \multicolumn{3}{|c|}{ Multivariable } & \multicolumn{3}{|c|}{ Multivariable } \\
\hline MSS & 1,00 & $0,010^{*}$ & $389(137)$ & 1,00 & $0,011^{*}$ & 185(107) \\
\hline \multirow[t]{2}{*}{ MSI } & $0,46(0,25-0,84)$ & & $73(13)$ & $0,33(0,14-0,78)$ & & $20(6)$ \\
\hline & \multicolumn{3}{|c|}{ Univariable } & \multicolumn{3}{|c|}{ Univariable } \\
\hline Bata-catenin low & 1,00 & 0,056 & $251(96)$ & 1,00 & 0,063 & 112(72) \\
\hline \multirow[t]{2}{*}{ Bata-catenin High } & $0,75(0,56-1,01)$ & & $276(86)$ & $0,73(0,52-1,02)$ & & $122(66)$ \\
\hline & \multicolumn{3}{|c|}{ Multivariable } & \multicolumn{3}{|c|}{ Multivariable } \\
\hline Bata-catenin low & 1,00 & $0,031^{*}$ & $223(80)$ & 1,00 & $0,036^{*}$ & $98(60)$ \\
\hline Bata-catenin High & $0,70(0,51-0,97)$ & & $247(75)$ & $0,67(0,46-0,97)$ & & $113(57)$ \\
\hline
\end{tabular}

*Significant at the 0.05 level. **Significant at the 0.01 level. P-value from multivariable analysis adjusted for age (>/<=75 years), gender, T-stage (I-II, III, IV), N-stage $(0,1,2), M$-stage $(0,1)$, differentiation grade (high-intermediate vs low) and vascular invasion (+/-/unknown). Beta-catenin low $=$ beta-catenin score $0-2$. Beta catenin high = beta-catenin score $3-5$. 

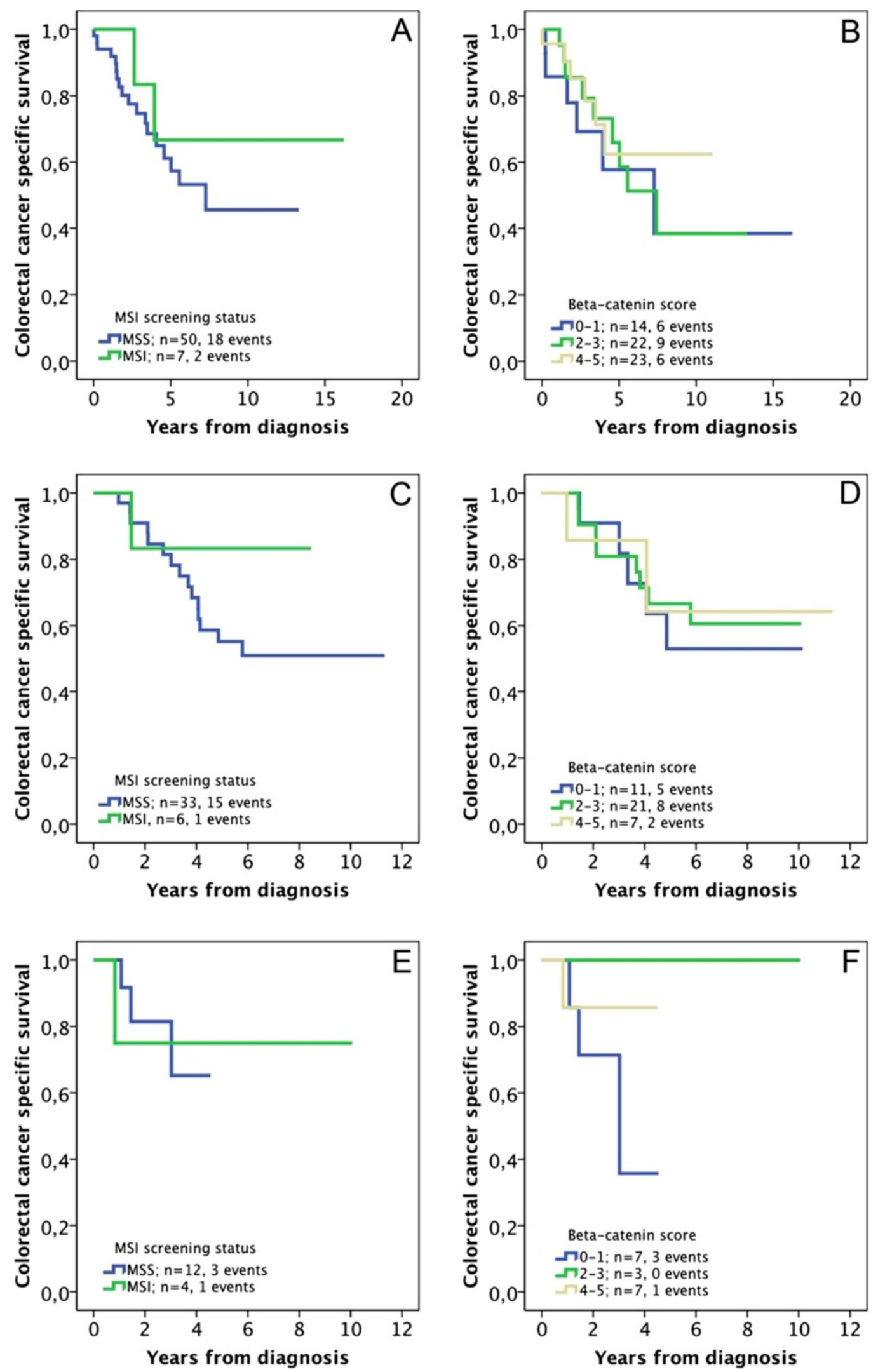

Figure 3 (See legend on next page.) 
(See figure on previous page.)

Figure 3 Associations between MSI screening status, beta-catenin expression and survival in stage III disease stratified according to adjuvant therapy. Kaplan-Meier analysis of colorectal cancer specific survival according to (A) MSI screening status and no adjuvant treatment (logrank $p=0.415)$, (B) Beta-catenin score and no adjuvant treatment (logrank $p=0.783)$, (C) MSI screening status and adjuvant FLV (5-fluorouracil + leucovorin ) (logrank $p=0.251$ ), (D) Beta-catenin score and adjuvant FLV (logrank $p=0.927)$, (E) MSI screening status and adjuvant FLOX (5-fluorouracil + leucovorin + oxaliplatin ) (logrank $p=0.871$ ) and (F) Beta-catenin score and adjuvant FLOX (logrank $p=0.336$ ).

expected, with no predilection for less advanced stages $[26,28]$. Notably, while the distribution of clinicopathological factors and survival from the disease did not differ between women and men [26], a recent study demonstrated sex-related differences in the associations of anthropometric factors with CRC risk by TNM stage and tumour location in the present cohort [49]. These findings provide further evidence for colorectal cancer being a disease in which the influence of sex hormones should not be underestimated. Therefore, the prognostic and treatment predictive value of investigative CRC biomarkers should always be considered in strata according to gender. Moreover, it will be of interest to investigate the influence of anthropometric, hormonal and life-style factors on risk of CRC defined by MSI status and betacatenin alterations in the present cohort.

Since the number of events was identical for CRCspecific and overall survival in patients with metastatic disease, as previously demonstrated [26], the use of CRC-specific survival as primary endpoint in the survival analyses in this study should be a reasonable surrogate for cancer-specific outcome. Moreover, similar survival trends were observed for 5-year overall survival.

\section{Conclusions}

In conclusion, the findings from this large cohort study demonstrate that MSI screening status in colorectal cancer is an independent prognostic factor, but not in localized disease, and does not predict response to adjuvant chemotherapy. Despite its inverse correlation with MSI status, we also observed a link between beta-catenin and prolonged survival from colorectal cancer. Associations of MSI and beta-catenin alterations with other investigative and clinicopathological factors were largely in line with the expected.

\footnotetext{
Abbreviations

CRC: Colorectal cancer; MSI: Microsatellite instability; MDCS: Malmö diet and cancer study.

\section{Competing interests}

The authors declare that no competing interests exist.

\section{Authors' contributions}

SW collected clinical data, evaluated the immunohistochemical stainings, performed the statistical analyses and drafted the manuscript. JB assisted with the collection of clinical data and helped draft the manuscript. BN constructed the TMAs and carried out all IHC stainings. KEL participated in the evaluation of the immunohistochemical staining. $\mathrm{KJ}$ conceived of the study, carried out the histopathological re-evaluation, evaluated the immunohistochemistry, and helped draft the manuscript. JE conceived of
}

the study, assisted with the collection of clinical data, and helped draft the manuscript. All authors read and approved the final manuscript.

\section{Acknowledgements}

This study was supported by grants from the Knut and Alice Wallenberg Foundation, the Swedish Cancer Society, the Gunnar Nilsson Cancer Foundation, Region Skåne and the Research Funds of Skåne University Hospital.

\section{Author details}

${ }^{1}$ Department of Clinical Sciences, Division of Pathology, Lund University, Skåne University Hospital, Lund 221 85, Sweden. Department of Clinical Sciences, Division of Oncology, Lund University, Skåne University Hospital, Lund 22185 , Sweden.

Received: 11 December 2012 Accepted: 13 January 2013

Published: 21 January 2013

\section{References}

1. Jemal A, Bray F, Center MM, Ferlay J, Ward E, Forman D: Global cancer statistics. CA Cancer J Clin 2011, 61:69-90.

2. Armaghany T, Wilson JD, Chu Q, Mills G: Genetic alterations in colorectal cancer. Gastrointest Cancer Res 2012, 5:19-27.

3. Pancione M, Remo A, Colantuoni V: Genetic and epigenetic events generate multiple pathways in colorectal cancer progression. Patholog Res Int 2012, 2012:509348.

4. Pino MS, Chung DC: The chromosomal instability pathway in colon cancer. Gastroenterology 2010, 138:2059-2072.

5. Al-Sohaily S, Biankin A, Leong R, Kohonen-Corish M, Warusavitarne J: Molecular pathways in colorectal cancer. J Gastroenterol Hepatol 2012, 27:1423-1431.

6. Half E, Bercovich D, Rozen P: Familial adenomatous polyposis. Orphanet Rare Dis 2009, 4:22.

7. Brabletz $T$, Jung A, Kirchner T: Beta-catenin and the morphogenesis of colorectal cancer. Virchows Arch 2002, 441:1-11.

8. Clevers H: Wnt breakers in colon cancer. Cancer Cell 2004, 5:5-6.

9. Klaus A, Birchmeier W: Wht signalling and its impact on development and cancer. Nat Rev Cancer 2008, 8:387-398.

10. Tetsu O, McCormick F: Beta-catenin regulates expression of cyclin D1 in colon carcinoma cells. Nature 1999, 398:422-426.

11. Shtutman M, Zhurinsky J, Simcha I, Albanese C, D'Amico M, Pestell R, BenZe'ev A: The cyclin D1 gene is a target of the beta-catenin/LEF-1 pathway. Proc Natl Acad Sci U S A 1999, 96:5522-5527.

12. Bondi J, Bukholm G, Nesland JM, Bukholm IR: Expression of nonmembranous beta-catenin and gamma-catenin, c-Myc and cyclin D1 in relation to patient outcome in human colon adenocarcinomas. APMIS 2004, 112:49-56.

13. Cheah PY, Choo PH, Yao J, Eu KW, Seow-Choen F: A survival-stratification model of human colorectal carcinomas with beta-catenin and p27kip1. Cancer 2002, 95:2479-2486.

14. Morikawa T, Kuchiba A, Yamauchi M, Meyerhardt JA, Shima K, Nosho K, Chan AT, Giovannucci E, Fuchs CS, Ogino S: Association of CTNNB1 (betacatenin) alterations, body mass index, and physical activity with survival in patients with colorectal cancer. Jama 2011, 305:1685-1694.

15. Drebber U, Madeja M, Odenthal M, Wedemeyer I, Monig SP, Brabender J, Bollschweiler E, Holscher AH, Schneider PM, Dienes HP, Vallbohmer D: betacatenin and Her2/neu expression in rectal cancer: association with histomorphological response to neoadjuvant therapy and prognosis. Int J Color Dis 2011, 26:1127-1134.

16. de la Chapelle A, Hampel $\mathrm{H}$ : Clinical relevance of microsatellite instability in colorectal cancer. J Clin Oncol 2010, 28:3380-3387. 
17. Boland $C R$, Goel A: Microsatellite instability in colorectal cancer. Gastroenterology 2010, 138:2073-2087. e2073.

18. Ribic CM, Sargent DJ, Moore MJ, Thibodeau SN, French AJ, Goldberg RM, Hamilton SR, Laurent-Puig P, Gryfe R, Shepherd LE, et al: Tumor microsatellite-instability status as a predictor of benefit from fluorouracil-based adjuvant chemotherapy for colon cancer. N Engl J Med 2003, 349:247-257

19. Kim GP, Colangelo LH, Wieand HS, Paik S, Kirsch IR, Wolmark N, Allegra CJ: Prognostic and predictive roles of high-degree microsatellite instability in colon cancer: a National Cancer Institute-National Surgical Adjuvant Breast and Bowel Project Collaborative Study. J Clin Oncol 2007, 25:767-772

20. Barratt PL, Seymour MT, Stenning SP, Georgiades I, Walker C, Birbeck K Quirke P: DNA markers predicting benefit from adjuvant fluorouracil in patients with colon cancer: a molecular study. Lancet 2002, 360:1381-1391.

21. Samowitz WS, Curtin K, Ma KN, Schaffer D, Coleman LW, Leppert M, Slattery ML: Microsatellite instability in sporadic colon cancer is associated with an improved prognosis at the population level. Cancer Epidemiol Biomarkers Prev 2001, 10:917-923.

22. Popat S, Hubner R, Houlston RS: Systematic review of microsatellite instability and colorectal cancer prognosis. J Clin Oncol 2005, 23:609-618.

23. Tajima A, Hess MT, Cabrera BL, Kolodner RD, Carethers JM: The mismatch repair complex hMutSa recognizes 5-fluorouracil-modified DNA: Implications for chemosensitivity and resistance. Gastroenterology 2004, 127:1678-1684.

24. Issa JP: CpG island methylator phenotype in cancer. Nat Rev Cancer 2004, 4:988-993.

25. Curtin K, Slattery ML, Samowitz WS: CpG island methylation in colorectal cancer: past, present and future. Patholog Res Int 2011, 2011:902674.

26. Wangefjord S, Manjer J, Gaber A, Nodin B, Eberhard J, Jirstrom K: Cyclin D1 expression in colorectal cancer is a favorable prognostic factor in men but not in women in a prospective, population-based cohort study. Biol Sex Differ 2011, 2:10.

27. Berglund G, Elmstähl S, Janzon L, Larsson SA: The malmo diet and cancer study. Design and feasibility. Journal of internal medicine 1993, 233:45.

28. Larsson A, Johansson ME, Wangefjord S, Gaber A, Nodin B, Kucharzewska P, Welinder C, Belting M, Eberhard J, Johnsson A, et al: Overexpression of podocalyxin-like protein is an independent factor of poor prognosis in colorectal cancer. Br J Cancer 2011, 105:666-672.

29. Eberhard J, Gaber A, Wangefjord S, Nodin B, Uhlen M, Ericson Lindquist K, Jirstrom $\mathrm{K}$ : A cohort study of the prognostic and treatment predictive value of SATB2 expression in colorectal cancer. Br J Cancer 2012, 106:931-938.

30. Ogino S, Kawasaki T, Kirkner GJ, Yamaji T, Loda M, Fuchs CS: Loss of nuclear p27 (CDKN1B/KIP1) in colorectal cancer is correlated with microsatellite instability and CIMP. Mod Pathol 2007, 20:15-22.

31. Dahlin AM, Palmqvist R, Henriksson ML, Jacobsson M, Eklof V, Rutegard J, Oberg A, Van Guelpen BR: The role of the CpG island methylator phenotype in colorectal cancer prognosis depends on microsatellite instability screening status. Clin Cancer Res 2010, 16:1845-1855.

32. Jass JR, Biden KG, Cummings MC, Simms LA, Walsh M, Schoch E, Meltzer SJ, Wright C, Searle J, Young J, Leggett BA: Characterisation of a subtype of colorectal cancer combining features of the suppressor and mild mutator pathways. J Clin Pathol 1999, 52:455-460.

33. Nodin B, Johannesson H, Wangefjord S, DP OC, Ericson-Lindquist $K$, Uhlen $M$, Jirstrom K, Eberhard J: Molecular correlates and prognostic significance of SATB1 expression in colorectal cancer. Diagn Pathol 2012, 7:115.

34. Des Guetz G: Microsatellite instability does not predict the efficacy of chemotherapy in metastatic colorectal cancer. A systematic review and meta-analysis. Anticancer Res 2009, 29:1615-1620.

35. Elzagheid A: Nuclear $\beta$-catenin expression as a prognostic factor in advanced colorectal carcinoma. World J Gastroenterol 2008, 14:3866.

36. Chung GG, Provost E, Kielhorn EP, Charette LA, Smith BL, Rimm DL: Tissue microarray analysis of beta-catenin in colorectal cancer shows nuclear phospho-beta-catenin is associated with a better prognosis. Clin Cancer Res 2001, 7:4013-4020

37. Norwood MG, Bailey N, Nanji M, Gillies RS, Nicholson A, Ubhi S, Darnton رل Steyn RS, Womack C, Hughes A, et al: Cytoplasmic beta-catenin accumulation is a good prognostic marker in upper and lower gastrointestinal adenocarcinomas. Histopathology 2010, 57:101-111.
38. Wanitsuwan W: Overall expression of beta-catenin outperforms its nuclear accumulation in predicting outcomes of colorectal cancers. World J Gastroenterol 2008, 14:6052.

39. Martensson A, Oberg A, Jung A, Cederquist K, Stenling R, Palmqvist R: Betacatenin expression in relation to genetic instability and prognosis in colorectal cancer. Oncol Rep 2007, 17:447-452.

40. Lugli A, Zlobec I, Minoo P, Baker K, Tornillo L, Terracciano L, Jass JR: Prognostic significance of the wnt signalling pathway molecules APC, beta-catenin and E-cadherin in colorectal cancer: a tissue microarraybased analysis. Histopathology 2007, 50:453-464

41. Buob D, Fauvel H, Buisine MP, Truant S, Mariette C, Porchet N, Wacrenier A Copin MC, Leteurtre E: The complex intratumoral heterogeneity of colon cancer highlighted by laser microdissection. Dig Dis Sci 2012, $57: 1271-1280$

42. Brabletz T, Jung A, Hermann K, Günther K, Hohenberger W, Kirchner T: Nuclear Overexpression of the Oncoprotein $\beta$-Catenin in Colorectal Cancer is Localized Predominantly at the Invasion Front. Pathol Res Pract 1998, 194:701-704.

43. Horst D, Reu S, Kriegl L, Engel J, Kirchner T, Jung A: The intratumoral distribution of nuclear beta-catenin is a prognostic marker in colon cancer. Cancer 2009, 115:2063-2070.

44. Cheng $H$, Liang $H$, Qin $Y$, Liu Y: Nuclear beta-catenin overexpression in metastatic sentinel lymph node is associated with synchronous liver metastasis in colorectal cancer. Diagn Pathol 2011, 6:109.

45. Kawasaki T, Nosho K, Ohnishi M, Suemoto Y, Kirkner GJ, Dehari R, Meyerhardt JA, Fuchs CS, Ogino S: Correlation $\beta$-Catenin Localization with Cyclooxygenase-2 Expression and CpG Island Methylator Phenotype in Colorectal Cancer. Neoplasia 2007, 9:569-577.

46. Jass JR: Classification of colorectal cancer based on correlation of clinical, morphological and molecular features. Histopathology 2007,

50:113-130

47. Nosho K, Kawasaki T, Chan AT, Ohnishi M, Suemoto Y, Kirkner GJ, Fuchs CS Ogino S: Cyclin D1 is frequently overexpressed in microsatellite unstable colorectal cancer, independent of CpG island methylator phenotype. Histopathology 2008, 53:588-598.

48. Ogino S, Kawasaki T, Kirkner GJ, Ogawa A, Dorfman I, Loda M, Fuchs CS: Down-regulation of p21 (CDKN1A/CIP1) is inversely associated with microsatellite instability and CpG island methylator phenotype (CIMP) in colorectal cancer. J Pathol 2006, 210:147-154.

49. Brandstedt J, Wangefjord S, Nodin B, Gaber A, Manjer J, Jirstrom K: Gender, anthropometric factors and risk of colorectal cancer with particular reference to tumour location and TNM stage: a cohort study. Bio/ Sex Differ 2012, 3:23

doi:10.1186/1746-1596-8-10

Cite this article as: Wangefjord et al:: Associations of beta-catenin alterations and MSI screening status with expression of key cell cycle regulating proteins and survival from colorectal cancer. Diagnostic Pathology 2013 8:10.

\section{Submit your next manuscript to BioMed Central and take full advantage of:}

- Convenient online submission

- Thorough peer review

- No space constraints or color figure charges

- Immediate publication on acceptance

- Inclusion in PubMed, CAS, Scopus and Google Scholar

- Research which is freely available for redistribution 